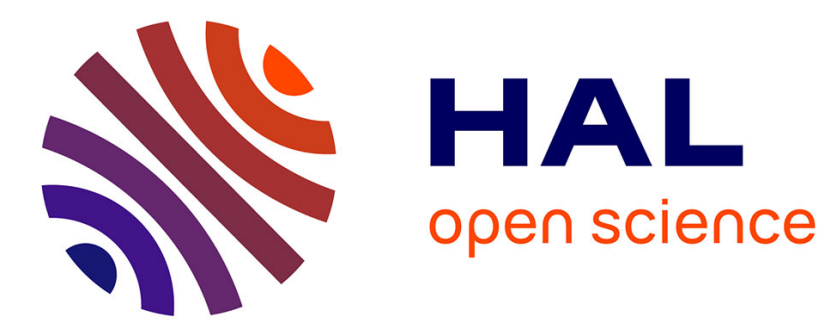

\title{
Unité et pluralité du vécu corporel en situation motrice
} Michel Récopé, Hélène Fache, Simon Boyer, Géraldine Rix-Lièvre

\section{To cite this version:}

Michel Récopé, Hélène Fache, Simon Boyer, Géraldine Rix-Lièvre. Unité et pluralité du vécu corporel en situation motrice. Movement \& Sport Sciences - Science \& Motricité, 2013, 1 (81), pp.37-47. hal-00984009

\section{HAL Id: hal-00984009 \\ https://hal.science/hal-00984009}

Submitted on 26 Apr 2014

HAL is a multi-disciplinary open access archive for the deposit and dissemination of scientific research documents, whether they are published or not. The documents may come from teaching and research institutions in France or abroad, or from public or private research centers.
L'archive ouverte pluridisciplinaire HAL, est destinée au dépôt et à la diffusion de documents scientifiques de niveau recherche, publiés ou non, émanant des établissements d'enseignement et de recherche français ou étrangers, des laboratoires publics ou privés. 


\title{
Unité et pluralité du vécu corporel en situation motrice
}

\author{
Michel Récopé, Hélène Fache, Simon Boyer, Géraldine Rix-Lièvre
}

Laboratoire ACTé-EA4281, Université Blaise Pascal, Clermont Université

Michel.Recope@univ-bpclermont.fr

\section{Résumé :}

Des insatisfactions issues du terrain de la formation en volley-ball nous conduisent à tenter de comprendre le vécu corporel des pratiquants. Une éthologie phénoménologique fondée sur des postulats énactifs permet de décrire l'activité de deux populations à partir de diverses régularités repérables. Deux micro-identités de pratiquants sont identifiées : chacune réfère à une cohérence et à un sens d'ordre global, qui ouvre et clôt un domaine d'interaction particulier lors des matchs, mais délègue la contextualisation et la régulation d'interactions plus spécifiques à d'autres registres perception-action. Nous proposons l'esquisse d'un modèle de la coordination de vécus corporels en situation motrice.

Mots clés : énaction ; éthologie phénoménologique ; micro-identité ; vécu corporel ; domaine d'interaction.

\section{Unity and plurality of corporeal lived experience in motor situation.}

\begin{abstract}
:
Dissatisfaction from the field of volleyball training lead us to try to understand the player's corporeal lived experience. A phenomenological ethology based on enactive assumptions is used to describe the activity of two populations from capture of various regularities. Two micro-identities are identified: each of them refers to a global coherence and sense, which open and close a peculiar domain of interactions during games, but delegate contextualization and regulation of more specific interactions to other action-perception registers. We propose a model for the coordination of corporeal lived experiences in ecological motor situation.
\end{abstract}

Key Words: enaction; ethological phenomenology; micro identity; corporeal lived experience; domain of interaction. 


\section{Unité et pluralité du vécu corporel en situation motrice}

\section{Des mobiles imposés par le terrain}

La question du vécu corporel s'est imposée à nous suite à des observations et insatisfactions issues du terrain de la formation en volley-ball. Ces dernières confirment un constat largement documenté par les praticiens (Marsenach et Léziart, 1991) mais qui n'a pas trouvé de réponse satisfaisante à ce jour. Elles concernent l'importante variabilité interindividuelle et la stabilité individuelle de la «présence active ${ }^{1}$ des volleyeurs lors des matchs. Nos analyses établissent que cette «présence active» est peu corrélée avec les critères suivants : l'âge; les qualités physiques; le volume de pratique antérieure; le niveau de pratique ; les savoir-faire spécifiques du volley-ball manifestés hors situation de jeu lors de tests de précision. Elle n'est pas non plus liée au cadre dans lequel s'opère la pratique (enseignement scolaire obligatoire et optionnel ; secteur associatif) ni à la motivation affichée hors situation.

De nombreux joueurs adoptent une "attitude de repos" (debout, statique, bras ballants) et n'en sortent que pour réagir lorsque le ballon arrive à leur proximité immédiate. Ces pratiquants les moins actifs sont peu performants et il est particulièrement difficile de remédier à leur passivité lors du processus enseignement-apprentissage. Or tant qu'ils demeurent peu actifs, ils ne progressent quasiment pas; en revanche, s'ils deviennent plus actifs, ce sont ceux qui progressent le plus. D'autres, peu nombreux, présentent souvent une "attitude de garde" (position d'affût, jambes semi-fléchies, buste légèrement penché vers l'avant, bras relevés, appuis dynamiques) et se livrent à des jaillissements intenses y compris lorsque le ballon n'est pas dirigé vers la position qu'ils occupent. Ces pratiquants s'avèrent être les plus performants et ils progressent en cours de formation. On observe des cas intermédiaires entre ces deux extrêmes qui nous serviront pour étayer nos propositions.

Cette différence de présence active traversant les contextes de jeu (exercices, formes jouées à effectif variable avec divers partenaires et adversaires) qui génère une inégalité croissante de performance et d'apprentissage a impulsé une question de recherche visant une formation plus pertinente. La perspective éthique recouvre un intérêt théorique : proposer une formation efficace présuppose, autant que possible, une compréhension et une problématisation des conduites des pratiquants. Notre conviction est qu'une approche du vécu corporel nécessite le recours à une phénoménologie de l'action en situation et que celle-ci implique une étude écologique de la situation motrice de l'individu dans un domaine particulier d'activité socioculturelle (Andrieu, 2010), ici le volley-ball : c'est ce que nous proposons d'illustrer en réduisant l'étude au rapport qu'entretiennent en acte les pratiquants ${ }^{2}$ avec le jeu et les situations du jeu.

\section{Position théorique}

Nous admettons, comme Berthoz et Petit (2006), que la saisie perceptive du monde est ancrée dans l'action d'un organisme vivant et qu'il y a solidarité constitutive du sentir, du percevoir et de l'agir. Comme eux, et comme le proposaient déjà Goldstein, Canguilhem et Merleau-Ponty, nous sommes attentifs à considérer la globalité des conduites et l'unité de l'organisme humain dans son environnement socioculturel. C'est pourquoi il nous faut

\footnotetext{
${ }^{1}$ L'expression «présence active » se veut, en première approximation, fidèle au sens commun désignant un dynamisme repérable par un observateur extérieur. L'objet de notre étude est précisément de dépasser ce sens commun.

${ }^{2}$ Le qualificatif de «pratiquants » souligne la restriction à ce seul aspect d'une activité assurément plus complexe, puisque les joueurs sont aussi des personnes, des membres d'un collectif, etc.
} 
considérer le vécu corporel (le Leib phénoménologique) au plus près de l'action en sa totalité, et pas au sein de fragments d'actes isolés de leur ancrage existentiel.

Pour Canguilhem (1968), seul l'organisme individuel appréhendé dans sa totalité constitue une entité réellement organisée. Le concept d'individu désigne un statut ontologique caractérisé par un degré d'unité ontologique : l'individualité est une identité littéralement indivisible. C'est au niveau de l'organisme seulement que le concept d'individu est pertinent : c'est la convergence fonctionnelle des parties dans le tout qui constitue l'authentique «tout» (désignant ici l'unité ontologique) et fait que ce vrai « tout» est plus que la simple somme de ses parties. Seul un tel tout mérite le nom d'individu et seul l'organisme, compris comme niveau d'organisation, peut être conçu comme totalité. Les autres niveaux d'organisation (cellule, famille, société, biocénose, etc.) ne constituent pas de véritables totalités. Pour Canguilhem, la société, par exemple, n'est pas une totalité suffisamment intégrée pour être considérée comme un authentique tout; son unité comme sa finalité lui viennent de l'extérieur tandis qu'elles sont immanentes à l'organisme biologique, intrinsèquement pourvu de systèmes de régulation (Giroux, 2008). Canguilhem reconnaît très tôt le caractère autopoiétique de l'activité organique. L'existence d'un organisme consiste à se proposer luimême aux choses, selon certaines orientations qui lui sont propres, selon ses normes propres d'appréciation des situations. En tant que vivant, l'homme n'échappe pas à la loi générale des vivants. Le milieu propre de l'homme c'est le monde de sa perception, c'est-à-dire le champ de son expérience pragmatique où ses actions, orientées et réglées par les valeurs immanentes aux tendances, découpent des objets qualifiés, les situent les uns par rapport aux autres et tous par rapport à lui. En sorte que l'environnement auquel il est censé réagir se trouve originellement centré sur lui et par lui. On ne peut comprendre son action sans faire appel à ses comportements privilégiés issus de ses normes propres, permettant seules de rendre compte de ses comportements privilégiés dans des situations analogues (1952).

Cette proposition est confirmée par la perspective énactive, qui conçoit l'organisme comme identité qui s'auto produit et s'auto régénère en permanence dans et par ses échanges avec son environnement extérieur, produisant par ses interactions un soi cognitif et son monde adéquat: "les interactions ont une pertinence pour -et des conséquences sur- l'identité unitaire [...] qui est la source des valeurs informationnelles, intentionnelles ou sémantiques » (Varela, 2010, 52). La cognition est fondée sur la nature biologique des organismes, sur leurs actions et leur expérience : il y a expérience dès qu'un organisme vivant constitue une unité intégrative s'incarnant dans un univers cognitif complexe et formant un centre de perspective et d'activité particulier sur le monde. Pour Varela (1998), cette expérience est une forme de conscience sans réflexion, un vécu in situ et in actu qui constitue un énorme background. Il la distingue de l'expérience réflexive ou expérience de se référer à son propre vécu.

La cognition est incarnée: les structures cognitives dépendent des types d'expérience qui découlent du fait d'avoir un corps doté de diverses capacités sensori-motrices, elles émergent de schèmes sensori-moteurs récurrents qui permettent à l'action d'être guidée par la perception. Ces structures sont l'émergence d'un niveau global à partir d'un ensemble de variables et de processus locaux en interdépendance. Si elles s'inscrivent dans un contexte biologique, puis psychologique et culturel plus large, elles sont individualisées, et non prédonnées: il n'y a pas d'a priori, pas de syntaxe formelle régissant nos façons de catégoriser les éléments du monde (Penelaud, 2010). Il en résulte que la cognition est caractérisée par sa créativité adaptative débouchant sur une multiplicité de voies évolutives, seulement possibles parce que satisfaisant une seule exigence, à savoir l'exigence suprême de viabilité (la conservation de l'organisation vivante, sa non désintégration). Elle considère qu'il y a émergence de différents modes adaptatifs identitaires indissociables d'une diversité d'Umwelts, c'est-à-dire de mondes phénoménaux incommensurables car non superposables les uns aux autres. 
Il ressort de cet ensemble de propositions une conséquence radicale : chaque identité instaure une forme de cohérence globale caractérisée par une clôture des interactions possibles et du champ d'expérience.

\begin{abstract}
«Seule une petite partie des dynamiques environnementales prend le statut de perturbation au sein du domaine de pertinence propre à tel organisme. Toutes les autres interactions envisageables échappent à ses possibilités d'expérience. Seul ce qui influe sur l'état d'équilibre de l'organisme apparaît comme réel, précisément parce qu'il a cette influence. Toute interaction avec le monde a donc toujours, pour l'organisme, une signification existentielle. Cette interaction s'opère donc toujours sur un fond de valeur préfigurant de manière prototypique les qualités que le monde prendra. La perspective d'un organisme affirmant son identité susceptible d'être menacée établit une nouvelle vision du monde : il s'agit en permanence d'un monde de valeurs. Posséder un monde signifie d'abord et avant tout avoir affaire à des valeurs produites au sein du processus par lequel l'organisme affirme son identité » (Weber \& Varela 2002, 118).
\end{abstract}

Enfin, la connaissance est située, contextuelle : elle se construit à partir des actions dans de petits domaines localisés, d'ampleur et de complexité variables selon le règne animal. Chez l'homme, cela aboutit à ce que l'identité de l'organisme fait place, au cours de l'histoire personnelle, à l'émergence de multiples micro-identités et micro-mondes correspondants, vécus et éprouvés chacun au sein d'un des nombreux contextes d'activité socioculturelle de la vie quotidienne (Varela, 2010). Ceci ouvre à la question des micro-identités qui ne réfèrent pas directement à la conservation de la vie, mais à celle de nos modes de vie déterminés par nos normes et comportements préférentiels (Di Paolo, 2005). Il s'agit de formes supérieures d'identités autonomes au-delà du soi organique, assurant une régulation normative des interactions référée à des intérêts et préoccupations pratiques et orientant notre engagement sensé dans le monde. L'espace du sens s'ancre dans la capacité à distinguer entre ce qui est pertinent et ce qui ne l'est pas, ce qui est bon et mauvais en référence aux normes autogénérées qui sous-tendent tel mode de vie propre. Varela, Thompson et Rosch (1993) considèrent que la signification relève des schèmes de l'expérience corporelle et des structures préconceptuelles de notre sensibilité (à savoir notre mode de perception, notre manière de nous orienter et d'interagir avec les autres objets, événements ou personnes). L'activité et le vécu situés sont l'expression de la cohérence propre d'une micro-identité particulière: conformément au principe d'émergence, il y a intégration permanente des perceptions, des actions, des affects, des valeurs et des catégories. Nos actions effectives, sous leur forme la plus habituelle, expriment nos micro-identités déjà constituées; une phénoménologie de l'expérience ordinaire doit s'efforcer d' "identifier quelques uns des micro-mondes typiques que nous habitons tous les jours [...] sur la base de leur récurrence » (Varela, 2010, 225). Le repérage des récurrences est donc une exigence méthodologique : «quel que soit le type de système autonome que nous étudions, nous ne pouvons l'aborder qu'à partir de certaines régularités de son comportement, qui sont intéressantes pour nous, observateurs extérieurs, parce que nous avons un accès conjoint au fonctionnement du système et à ses interactions » (Varela, 1989, 10).

\title{
Méthodologie
}

Ce sont bien les interactions qu'un organisme ${ }^{3}$ régule normativement selon sa propre perspective, qu'on peut donc décrire à partir de régularités repérables. Signalons, qu'à notre connaissance, malgré les arguments et propositions ci-dessus évoqués, aucune étude n'a à ce jour entrepris une telle perspective de caractérisation d'une micro-identité à l'œuvre.

\footnotetext{
${ }^{3}$ Ici réduit à une micro-identité à l'œuvre au sein d'un domaine culturel et social particulier.
} 
Notre méthodologie relève d'une éthologie phénoménologique qui s'efforce de relier les aspects phénoménaux pour nous, observateurs extérieurs, et les phénomènes pour l'acteur. Il s'agit d'une perspective descriptive et comparative des actes, initiée par Thinès (1980), qui s'efforce de saisir les relations signifiantes de l'organisme avec son monde naturel spécifique (Umwelt). Nous l'appliquons pour viser une compréhension des types d'activité produits dans la vie ordinaire des hommes (Le Blanc, 2002) dans leur milieu propre, défini comme le monde de leur perception, c'est-à-dire le champ de leur expérience pragmatique (Canguilhem, 1952). Nous abordons le vécu corporel à partir d'une description des manifestations phénoménales des actions au sein d'un espace de visibilité publique : celui de l'actualisation du corps au dehors durant les situations objectives du jeu. "Sans le corps actif rien ne nous serait donné à connaître » (Berthoz et Petit, 2006, 170) : c'est pourquoi «nous efforcer de penser le «corps vécu », le «vivre incarné », du dedans, intrinsèquement » (Richir, 1993, 8) présuppose de partir du corps au dehors.

Nous procédons à des enregistrements audiovisuels de l'activité des volleyeurs ${ }^{4}$ en match : ils sont la trace permettant la description des actions, mais également le support des entretiens d'autoconfrontation (Theureau, 1992) visant à resituer les joueurs au plus près de leur pratique en situation. Deux types de matériaux ont été recueillis :

- relatifs à la pratique in situ, ce sont les comportements enregistrés par une caméra lors des matchs. Elle a été positionnée dans le prolongement du filet, en plan fixe et en contrebas, avec un objectif grand angle permettant d'enregistrer en continu les actes des joueurs étudiés tout en visionnant l'ensemble du terrain, des protagonistes et des circonstances du jeu. Ces matériaux sont analysés par le chercheur au cours d'une procédure d'observation systématique. Il s'agit de décrire le plus finement possible les comportements typiques des acteurs et les situations du jeu au cours desquelles ils sont manifestés ;

- relatifs aux ressentis et aux significations a posteriori, il s'agit des verbatim recueillis au cours d'entretiens d'autoconfrontation menés dans les jours suivant les matchs. L'attention est ici focalisée sur les phénomènes pour les joueurs (sur ce qu'ils disent a posteriori de ce qui est donné à/par la perception, de ce qui est ressenti). Le pratiquant est confronté aux enregistrements de son match pour susciter une documentation de son vécu largement implicite, préréfléchi (Vermersch, 1999). Il est invité à expliciter, montrer et commenter certains événements pertinents pour lui (Theureau, 1992), par des questions ouvertes portant sur ses centrations (qu'est-ce qui se passe, pour toi, à ce moment-là ?), ses ressentis (comment tu te sens, là ?) et ses actes (qu'est-ce que tu fais ?). Chaque sujet pouvait à tout moment contrôler la lecture de la bande-vidéo, arrêter celle-ci, repasser tout ou partie d'une séquence. Nous avons opéré par des relances en écho à leurs propos, pour les inciter à les poursuivre ou les approfondir en évitant de les influencer, mais aussi de les cantonner dans un registre explicatif évaluatif justificatif ou interprétatif (Theureau, 1992). Chaque entretien, d'une durée d'une heure, a été intégralement retranscrit. L'ensemble des verbalisations recueillies a été traité.

Le traitement des matériaux accorde donc le primat aux régularités repérables : nous admettons une impossibilité méthodologique pour le chercheur d'accéder à la singularité de chacune des "expériences-événements » contingentes car elles sont enchâssées chacune dans un contexte singulier, jamais reproductibles à l'identique. C'est ce qui fonde le principe de la schématisation $^{5}$ que Bartlett évoque à propos du tennis : «quand je joue le coup, je ne produis

\footnotetext{
${ }^{4}$ Nous avons observé depuis une vingtaine d'années plusieurs centaines de pratiquants dans les divers cadres de pratique évoqués. Pour l'étude ici brièvement présentée, nous avons analysé 8 pratiquants initialement caractérisés de «peu actifs », 8 de « moyennement actifs » et 8 de « très actifs », tous participants volontaires.

${ }^{5}$ Le schema désigne l'organisation active issue des expériences. La détermination de nos actions par les schemas est la plus fondamentale dans tous les cas où l'on peut être influencé par notre passé et dans tous les domaines
}

[Tapez un texte] 
jamais, en réalité, quelque chose d'absolument nouveau, et je ne répète pas non plus l'intégralité d'un coup passé » $(1932,202)$. C'est aussi ce que confirme l'expression non paradoxale d'une «répétition sans répétition »(Bernstein, 1966). Il reste alors la possibilité, évoquée par Varela, de s'attacher à ce qui est commun à la diversité des expériences contingentes, c'est-à-dire à l'expérience-condensation, dont un certain partage intersubjectif semble plausible (Scheler, 1955). Nous visons ainsi une schématisation par le chercheur des vécus et des actions des pratiquants : pas une reproduction fidèle et intégrale de tel vécu ou de telle action en particulier mais, selon les termes de Revault d'Allonnes (1915), la constitution d'une épure dégageant des traits essentiels, pertinents, significatifs. Cette décision méthodologique est inséparable d'une posture théorique affirmant notre capacité de dégager des invariants sur la base de corrélations et de régularités (Berthoz et Petit, 2006).

\section{Principaux résultats}

Nos études ont repéré trois types de régularités à partir des matériaux. Elles sont relatives aux comportements in situ, aux circonstances du jeu dans lesquelles ils sont manifestés et enfin aux ressentis et significations exprimés a posteriori lors des entretiens à propos de ces comportements et circonstances. Les dernières ont été constituées par induction à partir de la documentation de nombreuses séquences particulières, le pratiquant se livrant à des explicitations situées de son expérience («là, je l'ai pas vu, le poteau ») ou à des généralisations spontanées («quand je joue, j'fais pas attention à la douleur »). En résulte une constitution progressive de catégories thématiques, par regroupement des propos en fonction de leur proximité sémantique. La mise en perspective de ces régularités constitue le fondement de nos résultats et interprétations schématiques ${ }^{6}$.

L'examen systématique de ces régularités aboutit à une spécification qualitative du domaine d'interaction des trois populations de pratiquants : dans les termes de Varela et al. (1993), ceci renvoie à divers modes d'adaptation définis par leur cohérence propre et qui demeure tant qu'elle est viable pour le sujet dans le domaine d'existence de ses interactions. Cette cohérence est émergente, globale : pour Foucault, « une description globale resserre tous les phénomènes autour d'un centre unique » $(1969,19)$. Nos schématisation portent sur la classe des bonnes et mauvaises actions, sur les situations du jeu qui sont pertinentes, sur les significations et ressentis documentés, selon des critères de nécessité et de satisfaction propres (Maturana et Varela, 1994). Nous identifions ainsi trois micro-identités typiques et leur micro-monde correspondant caractérisés par trois domaines d'interaction (Récopé et al, sous presse).

Nous résumons ci-dessous les principaux résultats, repris par la suite, relatifs aux descriptions de l'activité des deux populations de pratiquants les plus contrastées.

\section{Les traits principaux de la cohérence propre et du domaine d'interactions des pratiquants «peu actifs » sont :}

- une orientation de la tête vers le ballon et du corps face au filet lorsque le ballon est dans le camp adverse et lorsqu'un partenaire intervient sur le ballon. Ils sont régulièrement positionnés face au filet et au camp adverse et regardent les interventions des adversaires et des partenaires en gardant une posture de repos et par une transition directe entre une attitude de repos et une activation par projection des bras sur le ballon.

- le volley-ball est un jeu de position : jouer, c'est être positionné à un endroit du terrain et attendre, en attitude de repos (debout, détendu, bras ballants), que le ballon arrive à proximité, et c'est se replacer systématiquement sur sa base spatiale après une intervention.

(sport, littérature, etc.). Bartlett précise que les schemas sont dynamiques, en développement constant, car ils sont des cadres organisés susceptibles d'être remaniés en raison de nouvelles expériences.

${ }^{6}$ Pour une présentation détaillée des matériaux, résultats et interprétations de ces populations, voir Récopé et Fache (2010) et Fache (2011).

[Tapez un texte] 
- c'est un jeu de main : leur préoccupation pratique consiste à assurer un bon placement des mains au moment du contact avec le ballon. Une situation du jeu devient pertinente lorsqu'ils constatent que le ballon est dirigé vers eux, ce qui s'accompagne d'une préparation des mains : dans toutes les autres situations, il n'y a pas lieu d'intervenir ni de se déplacer.

- leurs interventions s'opèrent sous certaines conditions contextuelles cognitivement contrôlées : elles sont subordonnées à la direction du ballon vers eux et à une évaluation préalable des chances de réussir à jouer le ballon (s'il est trop loin, ou trop bas pour réussir le bon geste, ils renoncent à intervenir).

- ils sont insatisfaits quand ils échouent alors qu'ils estiment que les conditions d'interventions sur le ballon étaient favorables.

Leur domaine d'interaction est restreint. En effet, les circonstances du jeu font que le ballon arrive très rarement à proximité d'un joueur statiquement positionné sur sa base spatiale. De plus, les multiples conditions auxquelles ils soumettent leurs interventions les conduisent à toucher peu de ballons. Ils semblent prisonniers et victimes d'un paradoxe : la focalisation sur la phase finale du bon geste qu'ils cherchent à produire occulte les préparations, déplacements et ajustements posturaux nécessaires à la réussite. Enfin, l'ensemble des fonctions afférentes à la défense de son camp et au soutien des partenaires leurs sont étrangères. On comprend mieux dès lors pourquoi ils sont les moins actifs et les moins performants. De fait, lorsque la formation échoue à transformer leur modalité de «présence active », ils progressent très peu.

Selon nos interprétations, ils ont incorporé une norme prévalente d'intervention sur le ballon qui les focalise sur des formes strictement gestuelles de savoir-faire codifiés du volley-ball (la passe haute à deux mains et la manchette). La sensibilité renvoyant à la manière de nous orienter et d'interagir avec les autres objets, événements ou personnes (Varela et al. 1993), nous considérons que la micro-identité de ces pratiquants est caractérisée par une cohérence globale relevant d'une sensibilité à la proximité $d u$ ballon (ces pratiquants seront nommés SPB). C'est cette norme et cette sensibilité (Récopé et al., 2011) qui assurent la régulation normative et la clôture de leurs interactions.

Les traits principaux de la cohérence propre et du domaine d'interactions des pratiquants «très actifs » sont :

- une orientation systématique et instantanée du corps face au ballon pour l'ensemble des situations où le ballon est en jeu (à l'exception du service effectué par un partenaire).

- une «attitude de garde » régulièrement manifestée au moment où le serveur adverse contacte le ballon, et avant qu'un attaquant adverse joue la balle.

- de fréquents jaillissements défensifs instantanés opérés dans le temps même de la frappe d'attaque adverse et dès qu'un partenaire effectue une intervention maladroite créant ainsi un incident de construction du jeu, et ce quelles que soient la position occupée sur le terrain, la direction de la trajectoire du ballon, la distance qui sépare le joueur du ballon.

- la poursuite de tentatives défensives effrénées pour toucher le ballon suite à un incident de construction du jeu produit par l'intervention d'un partenaire. Ces actes de non renoncement donnent parfois lieu à des chutes ou impacts avec des éléments matériels (sol, poteau, mur, gradins, corps d'un partenaire, etc.)

- des manifestations de frustration suite à une perte du point sur balle non défendue, immédiatement après que le ballon soit tombé directement dans leur camp sur un service ou une attaque adverse.

- un sentiment de plaisir intense accompagnant la réussite d'une intervention défensive personnelle.

- un sentiment de profonde insatisfaction lorsqu'un partenaire s'avère régulièrement coupable d'inactivité défensive. 
Pratiquer le volley-ball, c'est d'abord et avant tout éviter la rupture de l'échange ${ }^{7}$ au détriment de leur équipe et la provoquer au détriment de l'adversaire. Le déroulement du jeu est de bout en bout traversé par cet enjeu d'opposition. Leurs comportements attestent une proximité ${ }^{8}$ permanente à l'égard du ballon, mais s'ils s'approchent spatialement de celui-ci, c'est pour s'éloigner existentiellement d'un événement : il faut absolument que le ballon ne tombe pas par terre. Ces pratiquants évaluent l'ensemble des situations du jeu en fonction de leur caractère plus ou moins menaçant au regard de cet enjeu, sachant que toute situation est comme destinée à devenir dangereuse. Cette préoccupation pratique prend le pas sur toute considération liée aux aspects spatiaux, temporels, gestuels, organisationnels et d'intégrité corporelle du contexte d'intervention.

Leur domaine d'interaction est ample : redoutant la survenue d'un événement, ces pratiquants s'efforcent de le pré-parer et déploient une activité débordante, non contenue, pour s'en défendre. Ils sont intéressés par tous les moyens et tentatives permettant d'y parvenir: l'empan temporel et spatial, le caractère foisonnant et intensif de leurs déplacements et tentatives font qu'ils interviennent fréquemment dans le cours du jeu. Ils ne sont pas asservis à une stricte répartition des fonctions au sein du collectif et interviennent parfois dans l'urgence à la place d'un partenaire alors que jouer le ballon relève de la responsabilité de ce dernier. Ils se révèlent les plus performants et qui plus est ils progressent en cours de formation.

Selon nos interprétations, ils ont incorporé une norme prévalente s'avérant être celle, informelle, qui a fondé le sport volley-ball (Récopé, Fache, 2010) en tant que construit culturel. Nous considérons que la micro-identité de ces pratiquants est caractérisée par une cohérence globale relevant d'une sensibilité à l'enjeu de rupture de l'échange (ces pratiquants seront nommés SER).

C'est cette sensibilité qui assure la régulation normative et la clôture de leurs interactions.

Les situations objectives du jeu laissent ainsi place à des situations pertinentes au sein du micro-monde propre, au regard des préoccupations pratiques qui déterminent les interactions pertinentes selon une perspective micro-identitaire. Mais cette caractérisation globale de l'activité et du vécu corporel doit s'accompagner d'une tentative d'appréhension plus fine.

\section{Vers un modèle de la coordination de vécus corporels :}

Pour Varela et al. (1993), la cognition opère au moyen d'un réseau global consistant en niveaux multiples de sous-réseaux sensori-moteurs interconnectés caractérisés chacun par son domaine local d'opérativité. Ceci est un principe des réalisations robotiques de Brooks (1987), impliquant différentes strates produisant chacune une activité composante, définie comme un schème d'interactions spécifiques avec le monde, contribuant à l'activité d'ensemble. Pas de lieu unique, pas d'instance centrale délivrant une présentation du monde au sens traditionnel: chaque strate connecte les aspects du monde et de l'action qui sont pertinents pour les interactions relevant de son champ assimilateur. Une organisation analogue avait déjà été proposée par Reed (1982) : les actions ne sont pas des unités discrètes mais des relations imbriquées impliquant diverses boucles perceptivo-motrices mutuellement ajustables; les composants des actions sont eux-mêmes des actions; lorsque plus d'un

\footnotetext{
${ }^{7} \mathrm{Au}$ volley-ball, chaque «échange » débute par un service et se termine lorsque le ballon touche le sol ou lorsqu'un joueur commet une faute, il y a alors rupture de l'échange.

${ }^{8}$ Cette proximité doit être pensée au sens de Barbaras (2008, 319). Il s'agit d'une « proximité d'investissement » qui transcende le seul ordre spatio-temporel. Cette proximité engage l'existence toute entière et fait intervenir une dimension que l'on peut qualifier d'affective. Est proche ce vers quoi je suis tourné, ce dans quoi mon existence est engagée, bref ce dans quoi je suis investi.
} 
composant est impliqué dans une action (comme c'est presque toujours le cas), aucun d'eux n'apparaît individuellement de façon évidente. Elle est récemment confortée par Visetti et Rosenthal (2006). Soucieux d'une approche du sens qui ne dissocie pas a priori les registres de la perception, de l'action et de la connaissance, ces auteurs affirment qu'il faut reconnaître une diversité de phases et de processus d'organisation coexistant dans la réalisation de l'action. L'organisme, en tant qu'unité cohésive et projective agissant selon certaines dispositions pratiques typiques, est à la fois la source et l'effet de la cohésion de ces processus. Ils proposent de distinguer et de relier une diversité de processus constitutifs de l'expérience, laquelle ne se différencie et ne s'individue que progressivement (à l'échelle d'un temps très bref dans le déroulement de cette expérience) sur les versants de l'action comme de la perception. Ainsi, la question du sens implique celle des divers niveaux de sens impliqués.

Ces arguments plaident pour un modèle de la coordination de processus fonctionnels et de «leur rapport d'insertion dans la totalité indivisible d'un comportement individuel» (Canguilhem, 2007, 50).

L'intérêt d'une modélisation ayant un potentiel de capture de différents plans ou registres d'organisation, sur la base d'une description concrète de l'activité, est récemment confirmé par Di Paolo (2010). Suite aux repérages de régularités, nos analyses aboutissent à une esquisse de modélisation de l'action et du vécu corporel en tant que coordination de quatre registres de couplage perception-action. Chacun d'entre eux établit ses propres événements informationnels pertinents et assure ses contributions motrices à l'activité d'ensemble. Au sein d'une organisation hiérarchisée opérant en tant que réseau de subordination fonctionnelle, un registre global détermine la cohérence d'ensemble en ouvrant un espace de sens dans lequel s'inscrivent les autres registres. Ce registre hiérarchique circonscrit le domaine des interactions dans lequel s'engage le pratiquant: ainsi, il instruit les autres registres tout en leur déléguant la production et le contrôle d'interactions spécialisées au service d'une activité cohésive. Une pluralité de vécus et d'activités orientés par un registre global caractériserait donc le tout complexe et organisé émergeant de ces modules interactifs.

\section{Le registre le plus global de l'activité et du vécu corporel: un cadre d'interactions organisées par un sens global et un corps sensible}

Jusqu'à présent le seul évoqué, il est la source et la référence de la cohésion des autres registres à l'œuvre car il instaure la micro-identité globale de chaque pratiquant et la sensibilité qui le fait s'orienter positivement ou négativement vers tel aspect du jeu : «c'est la marque originaire d'un sens, ou d'une valeur, dont la teneur est tout aussi bien sociale que sensible » (Visetti et Rosenthal, 2006, 112).

Correspondant à la source d'investissement de soi dans le monde ${ }^{9}$, il est responsable de la mobilisation des pratiquants (Récopé, Lièvre, Rix-lièvre, 2010) qui s'exprime au sein de domaines d'interaction d'ampleur et de nature différentes. Il constitue un espace de sens qui ouvre et clôt les interactions dans lesquelles ceux-ci s'engagent.

Ce registre réfère à des pratiquants plus ou moins concernés par les déplacements du ballon, intéressés à défendre, à anticiper, à se déplacer, ainsi qu'à la nature des ressentis, satisfactions et frustrations éprouvés. Ainsi, à l'inverse des SER, les pratiquants SPB demeurent statiques, en position de repos, debout, bras ballants aussi longtemps qu'ils n'ont pas constaté que le ballon arrive à leur proximité. Lorsque c'est le cas, ils mobilisent leurs bras pour le contacter, et déplacent seulement un appui pédestre à cette fin. Aucune partie de leur corps, autre que les appuis, ne touche jamais le sol, sauf chute involontaire, extrêmement rare.

Une formation efficace devrait s'attacher à la fois à comprendre la cohérence globale actuelle des pratiquants et à saisir les conditions permettant de favoriser l'émergence de la cohérence globale de type SER : l'intériorisation du volley comme sport saturé par un enjeu culturel

\footnotetext{
${ }^{9}$ Selon la belle expression suggérée par Pierre Pastré (communication personnelle).
} 
d'opposition ; l'intégration des différences de projet d'utilisation du ballon entre " jeu avec » (assurer une continuité de l'échange avec les partenaires) et «jeu contre » (rechercher au plus tôt la rupture de l'échange au détriment des adversaires) ; la distinction entre «balle cadeau » (ne provoquant pas de crise spatio-temporelle) et «balle méchante » (induisant une crise spatio-temporelle menaçant du danger de rupture et obligeant à s'employer); l'intention constante de se donner -et d'offrir aux partenaires- les meilleures conditions de contact et de visée et d'interdire aux adversaires de bonnes conditions de visée, voire la possibilité même de contacter le ballon.

Les acquisitions spécifiques de ce registre sont essentielles, car le développement de la SER s'accompagne d'une disponibilité corporelle, servie par une relation tonico-affective (Wallon, 1949) dont témoigne l'état de garde, et ouvre l'accès aux apprentissages relatifs aux autres registres. Ce registre ne suffit cependant pas pour embrasser la totalité de l'activité et du vécu corporel des pratiquants en situation : la contribution d'au moins trois autres registres plus spécialisés nous semble indispensable dans le jeu.

\section{Le registre de la contextualisation de l'activité et du vécu corporel : un cadre d'interactions organisées par un sens tactique et un corps de rationalité pratique}

Les situations du jeu et le contexte d'opposition sont in fine toujours singulières, contingentes et situées. Le sens tactique réfère à la mise en œuvre de solutions permettant d'harmoniser les possibles personnels et le contexte actuel du jeu. Ceci requiert des décisions d'intervention in situ des pratiquants, nécessaires parce que les déplacements aériens du ballon sont permanents et que les situations du jeu évoluent en temps réel. Elles sont prises en fonction de nombreuses connaissances ayant le statut de ressources pour l'action.

Sur ce plan encore, il y a une importante différence entre les pratiquants SPB et SER quant aux événements pertinents. Les études centrées sur cet aspect ne considèrent pas cette variable et procèdent généralement à une dichotomie expert/novice ou pratiquant perfectionnés/de haut niveau (Afonso et al., 2012).

Les SPB, dont le domaine d'interaction est restreint et centré sur un présent temporel, focalisent leur attention sur un seul type de situations pertinentes : celles où le ballon les sollicite directement, en vertu d'une préoccupation pratique : réussir à bien placer les mains pour le jouer. C'est l'arrivée du ballon qui les fait jouer : si le ballon tombe au sol parce qu'ils ne le jouent pas, c'est parce que l'adversaire a bien joué, ou parce qu'un partenaire a mal joué car il leur a fait une mauvaise passe. Les ajustements pertinents consistent à bien "préparer les mains » juste avant le moment du contact ; ils réclament avec insistance des indications sur le bon placement qui a, pour eux, valeur de moyen-clé à apprendre. Cette focalisation cohérente fait qu'ils sont en attente, non concernés par les déplacements du ballon tant qu'il n'est pas dirigé vers eux. Leur réaction tardive est souvent rapportée à de prétendues difficultés de lecture/d'appréciation des trajectoires du ballon, mais ce verdict signe un empirisme simple (Visetti et Rosenthal, 2006) : un corps naturalisé, lieu d'une juxtaposition de dispositifs sensori-moteurs disparates, isolé d'un espace de sens englobant et d'une activité couplante qui constitue le corps en même temps que son extériorité propre. Selon nos interprétations, la raison essentielle est ailleurs : en effet, s'intéresser tôt à la trajectoire du ballon n'est pertinent que si on a des raisons d'être intéressé à prévoir tôt son lieu de destination.

Or c'est le cas pour les pratiquants SER, caractérisés par l'empan temporel et situationnel large de leur domaine d'interactions. Leur préoccupation consiste à pré-parer un événement qu'ils ne peuvent pas sentir: être en retard, en crise spatio-temporelle et ne pas réussir, malgré une tentative effrénée, à toucher le ballon avant qu'il tombe au sol. D'ailleurs, pour eux, une situation de jeu favorable est une situation pas encore dangereuse, mais qui risque fort de le devenir. Ce vécu est le levier de l'anticipation, considérée non comme une qualité 
de lecture du jeu, mais comme une urgente et inquiète nécessité : anticiper, c'est pré-parer le risque d'être surpris, d'être mal placé, d'être en retard, en difficulté dans le futur immédiat des situations. Une synergie fonctionnelle est à l'œuvre : chercher des indices permettant de prévoir les circonstances; chercher à ajuster son placement pour être avant le ballon là où il arrivera ; chercher à se donner les possibilités de réagir avec une vitesse d'exécution optimale. Selon Nicolau, l'attitude de garde est relationnelle avant d'être une posture mécanique: « cette position est celle d'un joueur qui prévoit que l'adversaire peut le mettre en difficulté » $(1983,51)$.

Quand cet événement se produit, il est douloureusement vécu : ces pratiquants sont enclins à la réflexivité sur ce qu'il aurait fallu faire et ce qu'il faudrait faire pour s'en prémunir. Par exemple, l'un d'entre eux, joueur de niveau mondial, éprouve le besoin d'identifier les habitudes de jeu des meilleurs serveurs, ceux qu'il reconnaît comme pouvant le mettre en défaut. Il demande que soient réalisés des montages de films actualisés pour se livrer à une recherche minutieuse (de régularités entre leur lancer de balle et la direction que prend leur service ; du service «sûr » qu'ils utilisent en fin de set ou de match ; de la destination la plus fréquente de leurs services). Ceci facilite sa prévision et offre en outre des solutions de perturbation/dissuasion de ces serveurs, par un jeu de placements adéquats au moment même où celui-ci s'apprête à servir (indications d'ajustement à destination de ses partenaires pour surcharger la zone de destination probable du service).

Ce registre implique toutes les acquisitions permettant de réguler les décisions et solutions motrices pour mieux les contextualiser grâce à des enseignements tirés de son histoire. La cohérence propre des SPB assure une clôture très réductrice de ces interactions tactiques. Les apprentissages répondent en particulier à la volonté de ne pas faire de « cadeaux » à l'adversaire et consistent en l'établissement de rapports entre circonstances, modalités d'intervention et degré de réussite, rapports progressivement étendus aux possibilités personnelles (points forts/points faibles), à celles de son équipe et de l'équipe adverse.

\section{Le registre de la visée par déviation : un cadre d'interactions organisées par un sens d'efficacité mécanique et un corps instrument de la précision}

L'efficacité de chaque intervention sur le ballon dépend en volley-ball de la précision de la trajectoire produite suite au contact par déviation. Il est nécessaire d'exercer un contrôle gestuel suffisamment long sur le ballon pour produire les forces lui imposant la trajectoire souhaitée, ce qui suppose une intention de visée ainsi qu'une préparation de celle-ci avant même d'avoir contacté le ballon. Les apprentissages relevant de ce registre d'interactions réclament de nombreuses répétitions dans des conditions de placement corporel favorisant la visée.

Le statisme des pratiquants SPB ne permet pas de satisfaire ces conditions, du fait de l'absence de déplacement exploratoire avant le contact du ballon et d'une focalisation exclusive sur le placement des mains : l'image du bon geste se réduit à un instantané lors du contact. L'implication corporelle concerne essentiellement les membres supérieurs et les sensations corporelles pertinentes se circonscrivent aux mains. De fait, ils touchent peu de ballons. Quand ils le touchent, ils sont souvent mal placés car loin de lui et ils réagissent trop tardivement et explosivement pour pouvoir contrôler la direction du ballon joué. Tant qu'ils restent ainsi stimulés par l'arrivée du ballon, condamnés à des réactions, ils ne peuvent progresser que sur des aspects minimes liés à la réalisation du contact.

En revanche, les pratiquants SER, se déplacent tôt et vite pour éviter et réduire les crises spatio-temporelles. Focalisés sur la prévision des déplacements du ballon, ils cherchent à la fois à bien se placer et à se placer suffisamment tôt : en mesure de toucher plus souvent le ballon, dans des meilleures conditions d'intervention, ils peuvent mettre en œuvre et réguler 
les solutions gestuelles de visée par déviation. Ils sont de ce fait en mesure d'opérer les apprentissages spécifiques de ce registre: création d'un temps de repérage et de visée, incorporation de zones de visée, allongement des membres supérieurs pour appliquer des forces directionnelles sur le ballon, etc. Ces apprentissages reposent sur la construction progressive d'un bon compromis entre les exigences mécaniques de la déviation du ballon, les conditions spatio-temporelles de la situation et les caractéristiques anatomiques du corps humain. Ceci présuppose un pratiquant correctement placé : or les formateurs, même s'ils admettent que la qualité du placement détermine la qualité de l'intervention, ne s'intéressent pas assez aux raisons d'agir qui fondent la nécessité de bien se placer avant de jouer le ballon. Il y a aussi une activité et un vécu corporel d'interactions avec le ballon.

\section{Le registre postural de l'activité et du vécu corporel : un cadre d'interactions organisées par un sens kinesthésique et un corps sensitif}

Ce registre est celui de l'équilibration spécifique du volley-ball, indissociable des postures et des sensations permettant l'efficacité de la visée. Si la stabilité posturale est essentielle pour la précision des actions finalisées, elle ne peut être isolée des activités et vécus corporels organisés au niveau des précédents registres : le mouvement et la posture, dans ses rôles antigravitaire et d'interface entre le corps et son environnement, doivent être coordonnés dans le cours de la perception et de l'action (Massion et al, 2004).

Souvent minoré dans la formation, méconnu car il constitue l'arrière-plan permanent mais discret des interventions, il implique l'ensemble du corps. Sa fonction est d'autoriser des appuis efficaces, d'assurer la stabilisation des placements et de libérer les membres supérieurs pour qu'ils dirigent le ballon, autant d'aspects plus ou moins favorisés ou contrariés par l'histoire posturale de chaque pratiquant, par le rapport d'équilibration au sol qu'il a auparavant construit. Ce registre englobe l'ensemble des apprentissages spécifiques autorisant les régulations équilibratrices nécessaires aux déplacements, aux ajustements du placement et aux régulations des postures indispensables à toute visée. Les apprentissages consistent à construire des systèmes de sensations et de catégories sensitives permettant la stabilisation posturale et sa régulation, notamment par la création de systèmes d'appuis et de repères d'alignement entre le bassin et les appuis. Est visé un corps résistant à son poids et aux forces de réaction du sol, un corps se soumettant aux exigences de l'intervention et de la visée, disponible pour s'ajuster aux circonstances et pour réguler ses différents segments (par dissociations et coordinations) dans des conditions jamais identiques.

Nos observations en situation écologique attestent que l'activité organisée au sein de ce registre est fortement dépendante de la fréquence et de la vigueur des tentatives des pratiquants et des mouvements qu'elles impliquent dans les plans sagittal, frontal et transversal pour : se déplacer ; toucher des ballons difficiles à jouer (bas, hauts, latéraux, vers l'avant, vers l'arrière); chercher à concrétiser au mieux, y compris en cas de retard et d'éloignement, des conditions de visées satisfaisantes.

La cohérence propre des pratiquants SPB sollicite peu ces fonctions posturales et équilibratrices spécifiques du volley-ball. En effet, leurs déplacements pour aller vers le ballon sont rares et très limités (deux pas au plus), leurs ajustements équilibrateurs réduits à un appui pour compenser les déséquilibres produits suite à la projection des bras sur le ballon. Leur renoncement à jouer le ballon lorsqu'ils estiment ne pas pouvoir réussir le bon geste, ou que le ballon est trop loin ou qu'il n'est pas pour eux, fait qu'ils ne s'engagent pas dans des postures inédites, des tentatives impliquant un abaissement du bassin et des fentes latérales. Ils n'explorent donc pas de nouvelles sensations équilibratrices et ne mettent pas en péril leurs schémas posturaux habituels. En revanche, les pratiquants SER, en raison de leur mobilisation intense, des nombreuses tentatives opérées à partir d'une position jambes fléchies et sur la base d'un tonus musculaire élevé (deux composantes de leur état de garde), s'aventurent dans 
des solutions équilibratrices inédites, souvent jusqu'à la chute. Ils explorent spontanément de nouvelles formes d'appuis et de sensations, ce qui favorise et nourrit le remaniement de leurs schémas posturaux initiaux vers ceux spécifiquement requis pour la pratique du volley-ball. Leur investissement étant à la mesure du caractère insupportable d'une rupture de l'échange subie sur balle non défendue, on peut faire l'hypothèse que leurs jaillissements intenses et instantanés sont l'expression d'un tonus musculaire élevé : on sait que le tonus musculaire influe sur l'activité posturale, et qu'il est fonction de facteurs psychoaffectifs tels l'intention, la vigilance et la motivation (Ajuriaguerra et Angelergues, 1962 ; Bullinger, 1998). Or, ce tonus constitue l'une des composantes de la création d'appui et de l'application de forces sur la surface de support (Bouisset, 1991). Il existe des arguments philosophiques portant sur les relations entre élan, appui et mouvement : le mouvement volontaire est dirigé vers ou loin de certaines choses. Il nous permet de nous approcher des choses avec lesquelles nous souhaitons nous unir, ou de nous éloigner des choses qui nous menacent et dont nous devons nous défendre (Nogué, 1936). La dimension énergétique de la conduite ne renvoie pas seulement à des aspects biomécaniques et à des capacités strictement physiques, mais à un rapport existentiel à certains objets ou événements. La proximité d'investissement à l'égard du ballon qui caractérise les pratiquants SER les meut à s'approcher spatialement du ballon, mais c'est pour s'éloigner existentiellement d'une situation détestable (ballon au sol de son terrain) et atteindre une situation désirée (relever le ballon). Pour Nogué, l'élan est un effort tourné vers un objet, effort victorieux de la résistance et de l'inertie corporelle qui nous arrache du lieu que nous occupions ; il est solidaire d'un appui qui utilise la résistance pour progresser car il tend à immobiliser la partie du corps sur laquelle il s'exerce de façon à permettre au mouvement de progresser et de se déployer librement par ailleurs. Il y aurait bien solidarité entre le sens kinesthésique, c'est-à-dire les sensations indissociables du tonus, des appuis, des postures, des efforts et tentatives, et le sens global de la cohérence interne. Nos observations établissent d'ailleurs que de nombreux débutants SER déploient lors des déplacements une énergie débordante qui s'avère excessive au regard de leurs possibilités musculaires de résistance, d'appui et de leur capacité d'équilibration. Ils ne parviennent pas à s'arrêter en fixant des appuis, à conserver le bassin à l'intérieur du polygone de sustentation, donc, dans de nombreux cas, à stabiliser leur posture : ce sont alors les acquisitions permettant d'assurer une régulation et un contrôle de l'énergie mobilisée qui sont essentiellement requises.

Notre esquisse de modélisation de la coordination de vécus corporels, reposant sur une description concrète de l'activité des pratiquants, nous parait rendre compte, de manière certes partielle, d'aspects significatifs d'une unité intégrative, ici une micro-identité à l'œuvre, assurant un centre de perspective et d'activité sur le monde (Di Paolo, 2005). Un sens global requiert, pour son actualisation et sa satisfaction, la contribution de trois autres registres de sens : il ouvre un espace de sens micro-identitaire qui clôt les interactions dans lesquelles ceux-ci opèrent. La schématisation des deux populations de pratiquants révèle deux domaines d'interaction d'ampleur et de nature très contrastées, qui contiennent et circonscrivent phénoménalement deux espaces différents d'apprentissages possibles. C'est en raison de leur cohérence globale propre que les pratiquants peu actifs sont peu performants et apprennent peu. C'est cette cohérence micro-identitaire que devrait appréhender une formation fondée sur le vécu corporel des pratiquant pour susciter son évolution vers celle qui caractérise les très actifs.

\section{Conclusion}

Notre contribution à l'unité et la pluralité non paradoxales du vécu corporel de l'action et l'esquisse d'un modèle de coordination à l'œuvre sont issues du terrain des pratiques corporelles. 
Nos descriptions concrètes, opérées au moyen d'une éthologie phénoménologique de l'activité et du vécu corporel qui confronte les phénomènes pour le chercheur et les phénomènes pour l'acteur, rejoignent trois propositions de Merleau-Ponty (1942) : il y a coordination par le sens; une unité de sens irréductible est appréhendée; ce sens est immanent et incarné.

Notre modélisation impliquant différents registres confirme que «sans qu'ils soient séparés ou autonomisés, les territoires sont différenciés et jouissent d'une relative autonomie. La pénétration réciproque de toutes les parties supposées est le propre de tout organisme » (Dagognet, 1997, 110).

Ces propositions constituent à nos yeux un hommage adressé selon une perspective phénoménologique à Bernstein, qui affirmait le remarquable caractère structural et unitaire de l'action motrice (1984). Il y a lieu d'étendre à la question du sens son hypothèse d'une délégation d'opérations de régulation locale à des structures décentralisées qui assurent le contrôle d'interactions spécialisées.

Ajuriaguerra J. de, Angelergues R., (1962). De la psychomotricité au corps dans la relation avec autrui ; à propos de l'œuvre d'Henri Wallon, Evolution psychiatrique, 27, 13-25.

Afonso, J., Garganta, J., McRobert, A., Williams, A. M., Mesquita, I. (2012).The perceptual cognitive processes underpinning skilled performance in volley-ball. Journal of Sports Science and Medicine, 11, 339-345.

Andrieu, B. (2010). Philosophie du corps. Paris : Vrin.

Barbaras, R. (2008). Introduction à une phénoménologie de la vie. Paris : Vrin.

Bartlett, F. C. (1932). Remembering: a study in experimental and social psychology, Cambridge: Cambridge University Press.

Bernstein, N. (1967). The coordination and regulation of movements. London: Pergamon Press.

Bernstein, N. (1984). Trends and problems in the study of investigation of physiology of activity. In Whiting, H.T.A., Human motors actions: Bernstein Reassessed, Amsterdam: North-Holland, 441-466.

Berthoz, A., Petit, J.-L. (2006). Phénoménologie et physiologie de l'action. Paris : Odile Jacob.

Bouisset, S. (1991). Relation entre support postural et mouvement intentionnel : approche biomécanique. Archives Internationales de Physiologie, de Biochimie et de Biophysique, 99, 5, A77-A92.

Brooks, R. A. (1991). Intelligence without representation. Artificial Intelligence, 47, 1-3, 139159.

Bullinger, A. (1998). La genèse de l'axe corporel, quelques repères. Enfance, 51, 1, 27-35.

Canguilhem, G. (1968). Etudes d'histoire et de philosophie des sciences concernant les vivants et la vie. Paris : Vrin.

Canguilhem, G. (2002). Le problème des régulations dans l'organisme et dans la société.

Ecrits sur la médecine. Paris : Seuil.

Canguilhem, G. (2007). Le Normal et le Pathologique. Paris : PUF (1966).

Dagognet, F. (1997). Georges Canguilhem, Philosophe de la vie. Le Plessis-Robinson: Institut Synthélabo.

Di Paolo, E. A. (2005). Autopoiesis, adaptivity, teleology, agency. Phenomenology and the Cognitive Sciences, 4, 429-452.

Di Paolo, E. A. (2010). Robotics inspired in the organism. Intellectica, 53-54, 129-162. 
Fache, H. (2011). L'expérience corporelle des pratiquants de Volley-ball en situation. Phénomènes et structure des apparaissants. Sarrebrück: Éditions Universitaires Européennes.

Foucault, M. (1969). L'archéologie du savoir. Paris : Gallimard.

Giroux, E. (2008). N'y a-t-il de santé que de l'individu? In A. Fagot-Largeault, C. Debru, M. Morange, H. J. HAN (Eds.), Philosophie et médecine (174-177). Paris : Vrin.

Le Blanc, G. (2002). La vie humaine, Anthropologie et biologie chez Georges Canguilhem. Paris : PUF.

Marsenach, J., Léziart, Y. (1991). Séances d'EPS et pratique scolaire du volley-ball. In J. Marsenach (Ed.), J., EPS, quel enseignement? Paris : INRP.

Massion, J., Alexandrov, A., Frolov, A. (2004). Why and how are posture and movement coordinated? Progress in Brain Research, 143, 13-27.

Maturana, H. R., Varela, F. J. (1994). L'arbre de la connaissance. Paris : Addison-Wesley.

Merleau-Ponty, M. (1942). La structure du comportement. Paris : PUF.

Nicolau, H. (1983). Volley-ball., Luxembourg : Fédération Luxembourgeoise de Volley-ball.

Nogué, J. (1936). La signification du sensible. Paris : Aubier.

Penelaud, O. (2010). Le paradigme de l'énaction aujourd'hui. Plastir, 1, 18.

Récopé, M., Fache, H. (2010). La sensibilité incorporée des volleyeurs les plus «actifs ». In A. Berthoz \& B. Andrieu (Eds.), Le corps en acte (83-108). Nancy : PU de Nancy.

Récopé, M., Fache, H., Fiard, J. (2011). Sensibilité, conceptualisation et totalité [activitéexpérience-corps-monde], Travail et Apprentissages, 7, 11-32.

Récopé, M., Lièvre, P., Rix-Lièvre, G. (2010). The commitment of polar expedition members to a project. Project Management Journal, 41(3), 45-56.

Récopé, M., Rix-Lièvre, G., Fache, H., Boyer, S. (2013). La sensibilité à, organisatrice de l'expérience vécue. L. Albarello, J.-M. Barbier, E. Bourgeois et M. Durand (Dir.), Expérience, activité, apprentissage (111-133). Paris : PUF.

Reed, E. S. (1982). An Outline of a Theory of Action Systems. Journal of Motor Behavior, $14,2,98-134$.

Revault d'Allonnes, G. (1915). Le schématisme. Compte rendu de la 13ème session de l'Association Française pour l'Avancement des Sciences, Le Havre, 1914. Paris : Secrétariat de l'Association, 563-574.

Richir, M. (1993). Le corps. Essai sur l'intériorité. Paris : Hatier.

Scheler, M. (1955). Le formalisme en éthique et l'éthique matériale des valeurs. Paris: Gallimard.

Theureau, J., 1992. Le cours d'action : analyse sémiologique. Berne : Peter Lang.

Thinès, G. (1980). Phénoménologie et sciences du comportement. Liège : Mardaga.

Varela, F. (1989). Autonomie et connaissance. Paris : Seuil.

Varela, F. (1998). Entretien avec Francisco Varela. La Recherche, 308, 109-112.

Varela, F. (2010). El fenomeno de la vida. Santiago de Chile : J. C. Saez editor.

Varela, F., Thompson, E., Rosch, E. (1993). L'inscription corporelle de l'esprit. Paris : Seuil.

Vermersch, P. (1999). Pour une psychologie phénoménologique. Psychologie Française, 44(1), 7-19.

Visetti, Y.-M., Rosenthal, V. (2006). Les contingences sensorimotrices de l'énaction. Intellectica, 1, 43, 105-116.

Wallon, H. (1949). Les origines du caractère chez l'enfant. Paris : PUF.

Weber, A., Varela, F. (2002). Life after Kant: Natural purposes and the autopoietic foundations of biological individuality. Phenomenology and the Cognitive Sciences, 1, 97125. 